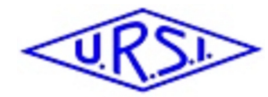

\title{
Assessing radiative transfer models trained by numerical weather forecasts using Sun-tracking radiometric measurements for satellite link characterization up to $\mathrm{W}$ band
}

\author{
Biscarini M.*(1), Montopoli M. ${ }^{(2)}$, Milani L. ${ }^{(1)}$, De Sanctis K. ${ }^{(3)}$, Di Fabio S. ${ }^{(4)}$, Madge K. ${ }^{(5)}$ Brost G. $^{(5)}$, Marzano F.S. ${ }^{(1)}$
}

(1) DIET, Sapienza University of Rome, Italy

(2) ISAC-CNR, Rome, Italy

(3) HIMET, L'Aquila, Italy

(4) CETEMPS, University of L'Aquila, Italy

(5) Air Force Research Laboratory, Rome, NY, USA

\begin{abstract}
Radio communications, and in particular Earth-to-satellite links, are worldwide used for delivering digital services. The bandwidth demand of such services is increasing accordingly to the advent of more advanced applications (e.g., multimedia services, deep-space explorations, etc.) thus pushing the scientific community toward the investigation of channel carriers at higher frequencies. When using carrier frequencies above $\mathrm{X}$ band, the main drawback is how to tackle the impact of tropospheric processes (i.e., rain, cloud, water vapor). This work assesses the joint use of weather forecast models, radiative transfer models and Sun-tracking radiometric measurements to explore their potential benefits in predicting path attenuation and sky noise temperature for slant paths at frequencies between $\mathrm{K}$ and $\mathrm{W}$ band, thus paving the way to the optimization of satellite link-budgets.
\end{abstract}

\section{Introduction}

The characterization of the Earth atmosphere is a crucial point for several microwave applications [1]. The knowledge of the atmospheric effects on the microwave signals is essential for the optimum design of an Earth-toEarth or a satellite-to-Earth link budget as well as for remote sensing purposes. A key quantity, when dealing with link budgets, is the level of signal-to-noise ratio registered at the receiving station for a certain carrier frequency. The signal-to-noise ratio is a direct consequence of two physically based frequency-dependent radiopropagation parameters that characterize the atmospheric state: the brightness temperature (BT) and the path integrated attenuation (PIA). The former takes into account the noise contribution of the whole atmosphere at the receiver of the receiving station, whereas the latter is responsible of the energy loss after the interaction between the transmitted signal and the atmosphere constituents along the signal path. Because of the atmospheric instability, both BT and PIA vary in space and time although some climatic regimes can be identified. For this reason, the space-time knowledge of BT and PIA is a fundamental task to optimize link budgets. To achieve this goal, traditional approaches, based on worldwide climatological statistics of radiopropagation parameters for the region of interest are used. Alternatively, more customized approaches aim at predicting BT and PIA by coupling outputs of a numerical weather forecast model (WFM) [2] and a radiative transfer model (RTM) [3], [4]. WFMs predict the atmospheric state in space and time, whereas RTMs [5] provide a numerical solution of the radiative transfer theory by converting the atmospheric state into radiopropagation variables (i.e., BT and PIA in our case).

In the context of deep-space satellite communications [6], we have already tested the coupling of WFM and RTM showing an improvement of the Ka-band radio link performance of about $20 \%$ in terms of yearly transferred data [3], [4] with respect to classical approaches. However, this result was supported by a simulated analysis since reference measurements were not available. To fill this gap, in this work we use reference measurements from a Suntracking microwave radiometer (ST-MWR) to assess the multi frequency performance of forecasted BT and PIA in clear sky, solid and liquid precipitation conditions obtained by the WFM and RTM chain [7]. ST-MWR is a groundbased radiometer that uses the Sun as a beacon to get simultaneous measurements of BT and PIA [7], [8].

In this work we investigate the frequency channels at 23.8, 31.4, 72.5 and $82.5 \mathrm{GHz}$. In addition, the WFM module is tested using radiosoundings and weather radar measurements. The test domain is the area of Rome, NY, at the Air Force Research Laboratory (AFRL) site. The WFM and RTM chain has the advantage to provide time series of forecasted BT and PIA consistent with the region and time of interest (where the WFM is run). The usability of forecasted BT and PIA can be questioned if their reliability is not accurately verified, as accomplished in this work. To this aim we consider two RTMs: $i$ ) a 1dimensional (1D) simplified RTM, developed in [9] and extended in [10], which is able to synthetically generate regional-driven radiopropagation parameters; ii) a 3dimensional (3D) RTM, fed by WFM, which is able to provide spatial and temporal evolution of radiopropagation parameters for a selected target domain [11]. 
The manuscript is organized as follows: section 2 describes models and measurements used for inter-comparisons between forecasts and reference observations, section 3 shows the quantitative comparison and section 4 draws the conclusions.

\section{Models and measurements}

Models and measurements discussed in this section refer to the test site in Rome, NY (USA) where the Air Force Research Laboratory (AFRL) is located (Figure 1b).

\section{$2.1 \quad$ Models}

Numerical weather forecast model (WFM). We adopt the Weather Research and Forecasting model [12] which is the state of the art of non-hydrostatic Numerical Weather Predictions. The model is initialized with global data analyses released every 6 hours from European Center for Medium-range Weather Forecast at $0.125^{\circ}$ resolution. The model works on two nested domains (Figure 1) with a resolution of $12 \mathrm{~km}(378 \times 596$ grid points $)$ and $4 \mathrm{~km}$ (96x148 grid points), respectively. The second domain includes the geographical areas of interest. The output of the model is the temporal evolution (with a release time of 1 hour) of the atmospheric state in the 3D-space in terms of vertical profiles of pressure, temperature, humidity, wind velocity, wind orientation and concentration of six atmospheric particles (i.e., cloud, rain, ice, snow, graupel and water vapor). We run the model collecting 1 year of simulations with time step of 1 hour from August 1, 2015 to July 31, 2016 in order to cover the period of measurements availability (cf. next section).

Radiative transfer models (RTMS). We consider two radiative transfer models. The first is a pseudo-3D model based on the Goddard Satellite Data Simulator Unit [11] that is able to convert the atmospheric state vector into a time series of BT and PIA as measured by a ground-based microwave radiometer at several off-zenith-angles (OZA) and frequencies. The model takes into account the gas absorption, the single scattering due to hydrometeors and five hydrometeor distributions (cloud, rain, ice, snow, graupel). The input atmospheric state vector, which can be provided by 3D-measurements (such as RAOB in clear sky) or predicted by a WFM, imposes the time resolution to the output radiopropagation variables. We adopt this 3D model driven by the WFM ('WFM+3DRTM') to simulate predicted BT and PIA. The second radiative transfer model that we consider is a sky noise Eddington model that was developed and described in [10]. It is a 1D-model (1DRTM) that gives an Eddington-based analytical approximation of the solution of the radiative transfer equation [9]. The 1DRTM ingests a synthetic clouds dataset for the random generation of seasonal-dependent and time-decorrelated meteorological variables at the desired frequency and OZA, with statistics driven by RAOB profiles in clear sky [10]. Both 3DRTM and 1DRTM simulations are obtained at several OZAs (i.e., $0^{\circ}$; $10^{\circ}, 20^{\circ}, 30^{\circ}, 36^{\circ}, 40^{\circ} 50^{\circ}, 54^{\circ}, 60^{\circ}, 70^{\circ}, 80^{\circ}$ ) for the frequencies at 23.8, 31.4, 72.5 and $82.5 \mathrm{GHz}$ where radiometric measurements are available.

\section{$2.2 \quad$ Measurements}

Sun-tracking microwave radiometer (ST-MWR). It is sited in Rome, NY, at the AFRL (lat. $43.21936 \mathrm{~N}$, lon. $75.408652 \mathrm{~W})$ [7]. Measurements are available at four frequency-channels $(23.8,31.4,72.5$ and $82.5 \mathrm{GHz})$ from May to October 2015 and for the whole year 2016 with a sampling time ranging from 2 to 5 seconds. Differently from conventional radiometers, this radiometer is able to provide simultaneous measurements of brightness temperature and attenuation in all-weather conditions exploiting the Sun as a stable radiance source [7], [8]. This is done by tracking the Sun path during its ecliptic motion and continuously switching the radiometer pointing angle from toward-the-Sun (tts) to out-of-Sun (oos) on the azimuthal plane. PIA value at a certain zenithal angle is retrieved through the difference between the two observations (tts-oos) at the same zenithal angle. This procedure allows compensating for any possible bias in the BT measurements. We exploit ST-MWR measurements for testing WFM+3DRTM and 1DRTM.

Radiosounding observations (RAOBS). Data are available from Albany, NY, observation site (lat. 42.69 N, lon. 73.83 $\mathrm{W}$, Figure 1b). RAOBs provide measurements of the vertical profiles of meteorological variables (pressure, temperature and humidity) at the observation site and are spaced in time of 12 hours in a day. We use RAOBs for testing the WFM in terms of 3D vertical profiles of humidity and temperature on the test period from August 1,2015 to July $31,2016$.

Weather radar (WR). Data are obtained from the Radar Archive of the National Centers for Environmental Information that includes the Next Generation Weather Radar System and Terminal Doppler Weather Radar networks. We use a large sample of weather-radar data for testing the WFM in terms of surface rain accumulations on a test period of 45 days in 2015.
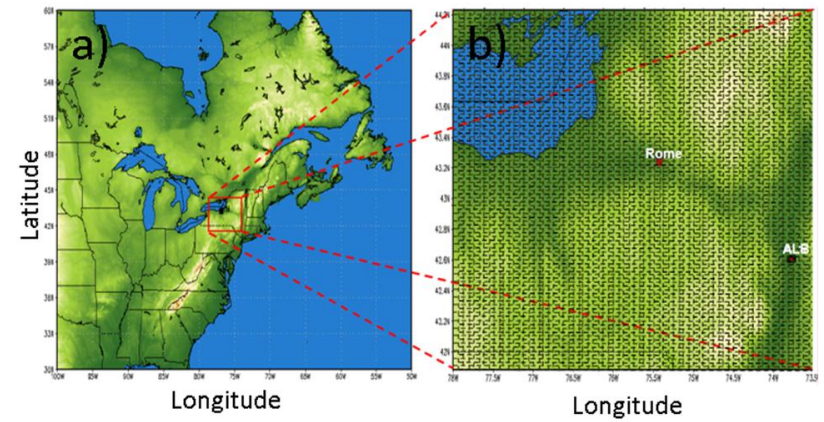

Figure 1. WFM domains: (a) domain 1 with $12 \mathrm{~km}$ resolution, (b) target domain with $4 \mathrm{~km}$ resolution and including Rome and Albany (ALB).

\section{Comparison results}

We have preliminarily tested the performance of the WFM (that feed the 3DRTM) on a volume centered on Albany site. We did it in terms of absolute humidity and 
temperature vertical profiles (from RAOB) and of surface rain accumulated in 1 hour (from weather radar). WFM test profiles refer to columns of base of $4 \times 4 \mathrm{~km}^{2}$ and height ranging from surface to approximatively $10-12 \mathrm{~km}$ whereas rain accumulations from weather radar are up-scaled to a regularly grid spaced by $5 \times 5 \mathrm{~km}^{2}$ to be more consistent with the WFM grid resolution. Quantitative results are in Table I and refer to the time periods described in subsection 2.2. The score indexes, reported in the table, are the correlation (Corr), bias, standard deviation (Std) and root mean square error (RMSE). The values highlight a good agreement between WFM and RAOBs, whereas for weather radar measurements the comparison is reasonably good considering the WFM "double penalty" due to raindistribution variability in space and time.

Table I. WFM validation with RAOB and weather radar measurements.

\begin{tabular}{l|cc|c}
\hline \hline & \multicolumn{2}{|c|}{ RAOB } & Weather radar \\
\cline { 2 - 4 } & $\begin{array}{c}\text { Absolute } \\
\text { humidity }\left(\mathbf{g} / \mathbf{m}^{\mathbf{3}}\right)\end{array}$ & $\begin{array}{c}\text { Temperature } \\
(\mathbf{K})\end{array}$ & $\begin{array}{c}\text { 1h-cumulated } \\
\text { rain (mm) }\end{array}$ \\
\hline Corr. & 0.97 & 0.99 & 0.42 \\
Bias & 0.07 & 0.17 & -0.09 \\
Std & 0.71 & 1.91 & 0.95 \\
RMSE & 0.71 & 1.92 & 0.95 \\
\hline \hline
\end{tabular}

After testing the WFM, the next step has been the comparison of the WFM+3DRTM outputs with ST-MWR measurements in Rome, NY, on the period of measurements availability. Figure 2 shows a timematching scatterplot in terms of BT for each of the four frequencies investigated. Due to the Sun-tracking operation mode, ST-MWR acquires measurements at continuously varying OZAs. On the other hand, WFM+3DRTM simulations are performed on the whole period at some fixed angles. In order to make a homogenous comparison, in Figure 2 we have selected WFM+3DRTM simulations and ST-MWR measurements at OZA $=54^{\circ}$, which is the most occurring angle during the ST-MWR antenna motion, considering a ST-MWR pointing tolerance equal to $+/$ $0.1^{\circ}$. Because of the differences in the time resolution between WFM+3DRTM and ST-MWR, which is 1 hour and few seconds, respectively, we have averaged ST-MWR measurements over 1 minute around each nominal hour of WFM+3DRTM simulations. So doing many samples are filtered out although the residual points reported in Figure 2 are enough to capture the data trend. We have applied the same matching strategy in terms of PIA (not shown for brevity). Error scores, for BT and PIA, are listed in Table II and show a general agreement between model and measurements. A larger data dispersion appears at high values of BT (Figure 2) where rainy events occur. In Table II, we notice a BT underestimation bias of WFM+3DRTM of the order of $6 \mathrm{~K}$ and $11 \mathrm{~K}$ depending on the frequency. Inaccuracies in the 3DRTM routines of radiative transfer can be responsible of these biases. Further source of errors can be ascribed to the slant path interpolation, implemented within 3DRTM, to extract BTs along the desired direction [11] or to possible calibration biases of the ST-MWR [8]. Additional investigations are ongoing exploiting traditional radiometric measurements to perform further and more robust comparisons.
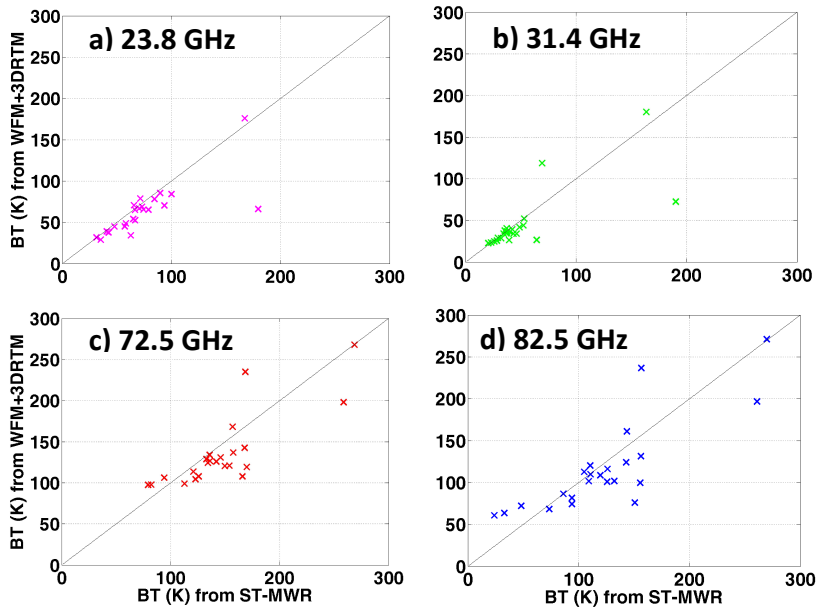

Figure 2. BT scatterplot after time matching of WFM+3DRTM vs ST$\mathrm{MWR}$ at $\mathrm{OZA}=54^{\circ}$ for the frequencies reported in each panel.

Table II. Error scores between WFM+3DRTM and ST-MWR

\begin{tabular}{l|l|c|c|c|c}
\hline \hline & Frequency (GHz) & $\mathbf{2 3 . 8}$ & $\mathbf{3 1 . 4}$ & $\mathbf{7 2 . 5}$ & $\mathbf{8 2 . 5}$ \\
\hline \multirow{4}{*}{ BT } & Corr. & 0.75 & 0.74 & 0.81 & 0.82 \\
& Bias (K) & -11.53 & -6.57 & -11.65 & -6.55 \\
& Std. (K) & 23.9 & 28.29 & 27.26 & 33.63 \\
& RMSE (K) & 26.54 & 29.04 & 29.64 & 34.26 \\
\hline \multirow{4}{*}{ PIA } & Corr. & 0.72 & 0.69 & 0.84 & 0.84 \\
& Bias (dB) & -0.23 & -0.14 & -0.47 & -0.32 \\
& Std. (dB) & 0.66 & 0.85 & 1.82 & 2.05 \\
& RMSE (dB) & 0.7 & 0.87 & 1.88 & 2.08 \\
\hline \hline
\end{tabular}

Eventually, Figure 3 and Figure 4 show a statistical comparison of both WFM+3DRTM and 1DRTM simulations with ST-MWR measurements in terms of BT channel correlation and BT-PIA correlation, respectively. These figures indicate that the WFM+3DRTM and 1DRTM are in good agreement and both well reproduce the overall trend of the ST-MWR measurements. Figure 3 confirms the WFM+3DRTM underestimation as well as that noted by 1DRTM at $23.8 \mathrm{GHz}$ for BT less than $75 \mathrm{~K}$. Figure 3 and Figure 4 point out the reduced dynamic (toward low values of BT and PIA) of both WFM+3DRTM and 1DRTM with respect to ST-MWR at $72.5 \mathrm{GHz}$ and even more at $82.5 \mathrm{GHz}$. Figure 4 highlights a saturation effect of PIA from ST-MWR at high BT values. This is due to the upper limit of the PIA retrieved by ST-MWR [8]: when heavy rain events occur, the atmosphere acts as a screen preventing the radiometer from receiving the Sun signal and causing a saturation of the retrieved PIA.

\section{Discussion and Conclusions}

In this work we have presented a test analysis to assess the performance of a model-based chain, using weather forecasts coupled with radiative transfer models to simulate brightness temperatures and path attenuations for a selected domain and time frame. We have used a four channel Suntracking microwave radiometer as reference to verify the model chain and radiosoundings and radar data to check the weather forecasts reliability. 

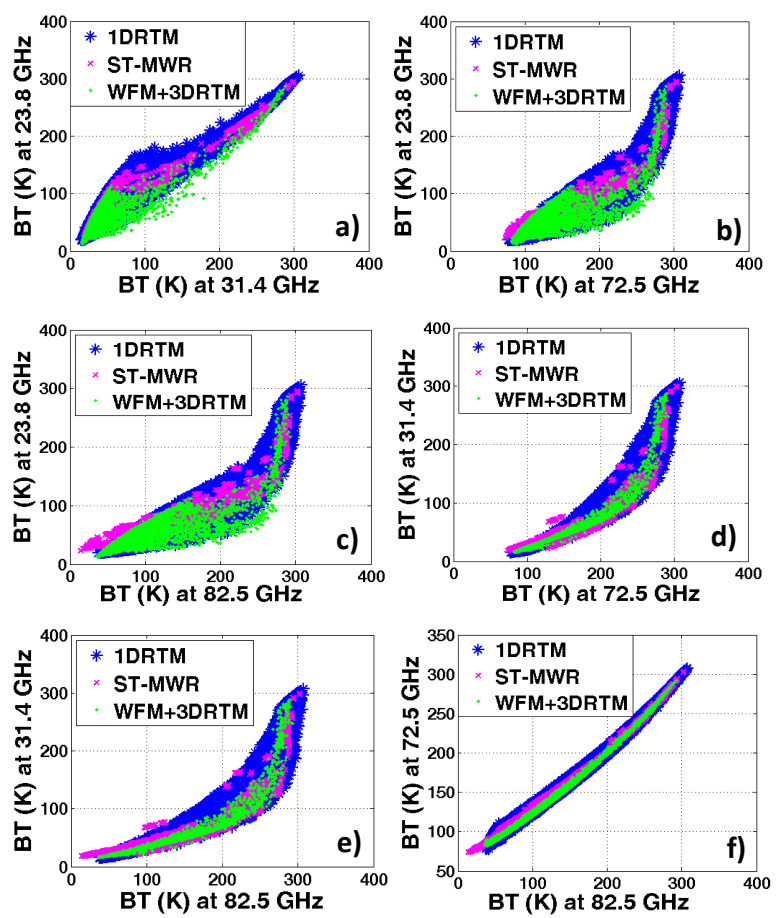

Figure 3. BT channel correlation of WFM+3DRTM and 1DRTM simulations vs ST-MWR measurements.
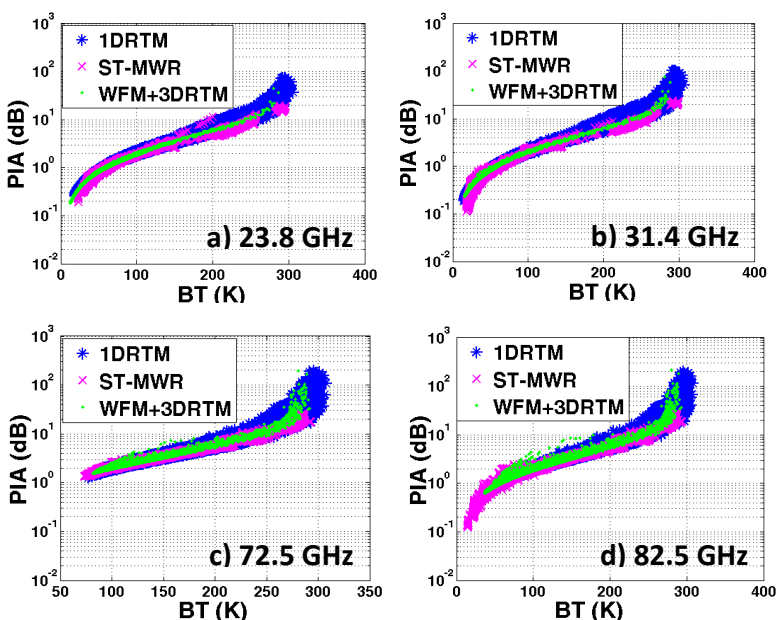

Figure 4. BT-PIA correlation of WFM+3DRTM and 1DRTM with STMWR at 23.8 GHz (a), 31.4 GHz (b), 72.5 GHz (c) and $82.5 \mathrm{GHz}(\mathrm{d})$.

Results show a good performance of weather forecast outputs in both clear sky and rainy conditions. On the other hand, simulations of brightness temperature and path attenuation may be biased when $3 \mathrm{D}$ radiative models are used and comparisons are performed considering Suntracking radiometers as reference. However, such biases can be compensated thus opening to the opportunity of using a coupled model chain (weather forecasts and radiative transfer routines) for radiopropagation applications. Indeed, the forecast of radiopropagation parameters can pave the way to a weather-forecast driven adaptation strategy of link budgets and can be exploited to make climatological statistics of radiopropagation parameters more robust. Moreover, the capability of the simpler 1DRTM to reproduce the statistical trend of Suntracking radiometric measurements adds reliability to this approach that can be a feasible solution to compensate the lack of measurements or the unavailability of more advanced radiative transfer models in a certain area and/or at specific frequencies. Future developments will be aimed to enrich the data comparison using traditional radiometers as reference and to further refine the $3 \mathrm{D}$ radiative transfer model simulations.

\section{References}

[1] G. Brussaard and P. A.Watson, "Atmospheric Modelling and Millimetre Wave Propagation". London, U.K.: Chapman \& Hall, 1995.

[2] M. O. García, N. Jeannin, L. Féral, and L. Castanet, "Use of WRF model to characterize propagation effects in the troposphere," in Proc. Eur. Conf. Antennas Propag. (EuCAP), Apr. 2013, pp. 1377-1381.

[3] M. Biscarini, F. S. Marzano, M. Montopoli, K. De Sanctis, L. Iess, M. Montagna, M. Mercolino and M. Lanucara, "Optimizing data volume return for Ka-band deep space links exploiting short-term radiometeorological model forecast", Antennas and Propagation, IEEE Transactions on, DOI: 10.1109/TAP.2015.2500910.

[4] M. Biscarini, M. Montopoli, F. S. Marzano, "Evaluation of High-Frequency Channels for Deep-Space Data Transmission Using Radiometeorological Model Forecast”, Antennas and Propagation, IEEE Transactions on, DOI: 10.1109/TAP.2017.2653420.

[5] F. T. Ulaby, R. K. Moore, and A. K. Fung,Microwave Remote Sensing, Active and Passive. Volume 1, Microwave Remote Sensing Fundamentals and Radiometry. Reading, MA: Addison-Wesley, 1981.

[6] F. Davarian, S. Shambayati, and S. Slobin, "Deep space Kaband link management and mars reconnaissance orbiter: Long term weather statistics versus forecasting," Proc. IEEE, vol. 92 , no. 12 , pp. 312-329, Nov./Dec. 2004;

[7] F. S. Marzano, V. Mattioli, L. Milani, K.M. Magde and G.A. Brost, "Sun-Tracking Microwave Radiometry: All-Weather Estimation of Atmospheric Path Attenuation at $\mathrm{Ka}-, \mathrm{V}-$, and W -Band", IEEE Transactions on Antennas and Propagation, vol. 64, 11, nov. 2016.

[8] V. Mattioli, L. Milani, K. M. Magde, G. A. Brost, and F. S. Marzano, "Retrieval of Sun Brightness Temperature and Precipitating Cloud Extinction Using Ground-Based SunTracking Microwave Radiometry", IEEE J. Sel. Topics Appl. Earth Observ. in Remote Sens., vol. 10, no. 7, 2017.

[9] F. S. Marzano, "Modeling antenna noise temperature due to rain clouds at microwave and millimeter-wave frequencies", IEEE Trans. Antennas and Propagat., vol. 54, pp. 13051317,2006

[10] V. Mattioli, F. S. Marzano, N. Pierdicca, C. Capsoni, and A. Martellucci, "Modeling and predicting sky-noise temperature of clear, cloudy, and rainy atmosphere from Xto W-band," IEEE Trans. Antennas Propag., vol. 61, no. 7, pp. 3859-3868, Jul. 2013.

[11] T Matsui., J. Santanello, J. J. Shi, W.-K. Tao, D. Wu, C. Peters-Lidard, E. Kemp, M. Chin, D. Starr, M. Sekiguchi, and F. Aires, "Introducing multisensor satellite radiancebased evaluation for regional Earth System modeling", Journal of Geophysical Research, 119, 8450-8475, doi:10.1002/2013JD021424, 2014.

[12] W.C. Shamarock et al., "A Description of the Advanced Research WRF Version 3", NCAR/TN-475+STR, 2008. 